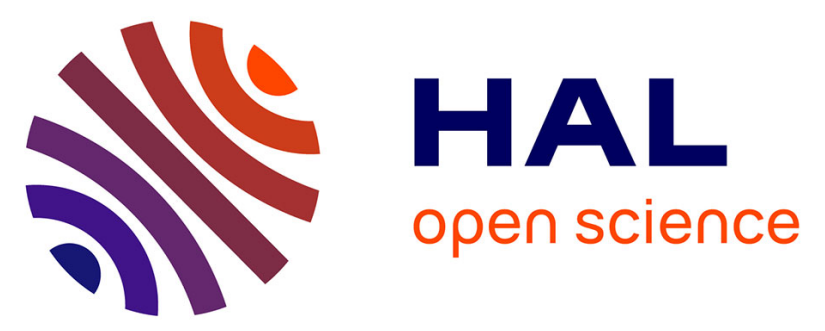

\title{
Homozygous and compound heterozygous mutations in the FBN1 gene: unexpected findings in molecular diagnosis of Marfan syndrome
}

Pauline Arnaud, Nadine Hanna, Mélodie Aubart, Bruno Leheup, Sophie

Dupuis-Girod, Sophie Naudion, Didier Lacombe, Olivier Milleron, Sylvie

Odent, Laurence Faivre, et al.

\section{To cite this version:}

Pauline Arnaud, Nadine Hanna, Mélodie Aubart, Bruno Leheup, Sophie Dupuis-Girod, et al.. Homozygous and compound heterozygous mutations in the FBN1 gene: unexpected findings in molecular diagnosis of Marfan syndrome. Journal of Medical Genetics, 2017, 54 (2), pp.100 - 103. 10.1136/jmedgenet-2016-103996 . hal-01670201

\section{HAL Id: hal-01670201 \\ https://hal.science/hal-01670201}

Submitted on 21 Dec 2017

HAL is a multi-disciplinary open access archive for the deposit and dissemination of scientific research documents, whether they are published or not. The documents may come from teaching and research institutions in France or abroad, or from public or private research centers.
L'archive ouverte pluridisciplinaire $\mathbf{H A L}$, est destinée au dépôt et à la diffusion de documents scientifiques de niveau recherche, publiés ou non, émanant des établissements d'enseignement et de recherche français ou étrangers, des laboratoires publics ou privés. 


\title{
Homozygous and compound heterozygous mutations in the FBN1 gene: unexpected findings in molecular diagnosis of Marfan syndrome
}

\author{
Pauline Arnaud, ${ }^{1,2}$ Nadine Hanna, ${ }^{1,3}$ Mélodie Aubart, ${ }^{3}$ Bruno Leheup, ${ }^{4}$ \\ Sophie Dupuis-Girod, ${ }^{5}$ Sophie Naudion, ${ }^{6}$ Didier Lacombe, ${ }^{6}$ Olivier Milleron, ${ }^{7}$ \\ Sylvie Odent, ${ }^{8}$ Laurence Faivre, ${ }^{9}$ Laurence Bal, ${ }^{10}$ Thomas Edouard, ${ }^{11}$ \\ Gwenaëlle Collod-Beroud, ${ }^{12}$ Maud Langeois, ${ }^{7}$ Myrtille Spentchian, ${ }^{7}$ Laurent Gouya, ${ }^{7}$ \\ Guillaume Jondeau, $^{2,7}$ Catherine Boileau ${ }^{1,2}$
}

\section{Correspondence to}

Professor Catherine Boileau, Département de Génétique, Hôpital Bichat, 46 rue Henri Huchard, Paris 75018, France; catherine.boileau@aphp.fr

\section{ABSTRACT \\ Background Marfan syndrome (MFS) is an autosomal- dominant connective tissue disorder usually associated with heterozygous mutations in the gene encoding fibrillin-1 (FBN1). Homozygous and compound heterozygous cases are rare events and have been associated with a clinical severe presentation. \\ Objectives Report unexpected findings of homozygosity and compound heterozygosity in the course of molecular diagnosis of heterozygous MFS and compare the findings with published cases.}

Methods and results In the context of molecular diagnosis of heterozygous MFS, systematic sequencing of the FBN1 gene was performed in 2500 probands referred nationwide. 1400 probands carried a heterozygous mutation in this gene. Unexpectedly, among them four homozygous cases (0.29\%) and five compound heterozygous cases $(0.36 \%)$ were identified (total: $0.64 \%$ ). Interestingly, none of these cases carried two premature termination codon mutations in the FBN1 gene. Clinical features for these carriers and their families were gathered and compared. There was a large spectrum of severity of the disease in probands carrying two mutated FBN1 alleles, but none of them presented extremely severe manifestations of MFS in any system compared with carriers of only one mutated FBN1 allele. This observation is not in line with the severe clinical features reported in the literature for four homozygous and three compound heterozygous probands.

Conclusion Homozygotes and compound heterozygotes were unexpectedly identified in the course of molecular diagnosis of MFS. Contrary to previous reports, the presence of two mutated alleles was not associated with severe forms of MFS. Although homozygosity and compound heterozygosity are rarely found in molecular diagnosis, they should not be overlooked, especially among consanguineous families. However, no predictive evaluation of severity should be provided.

\section{INTRODUCTION}

Marfan syndrome (MFS) is an inherited autosomaldominant disorder, with an estimated incidence of 1 in 5000 individuals, in which several systems (mainly cardiovascular, ocular, skeletal systems) are affected with great phenotypic variability.
Cardiovascular manifestations with thoracic aortic aneurysms or dissections are the most serious lifethreatening complications of the syndrome. Clinical variability is high in MFS and diagnosis is established according to revised Ghent nosology. ${ }^{12}$ The Ghent criteria comprise a set of major and minor manifestations in different body systems and are recognised by international experts. Heterozygous mutations in the FBN1 gene, encoding for fibrillin-1, an extracellular matrix protein, are found in the majority of patients with MFS. ${ }^{3}$ Besides severe neonatal cases with mutations between exons 24 and 32, no correlation between the type of mutation in the FBN1 gene and severity of the various manifestations of the disease could be made. ${ }^{4}$

The first heterozygous mutations in the FBN1 gene were published in $1991 .^{5}$ In 1994 , Karttunen et $a l^{6}$ described the first compound heterozygous case: a newborn boy with a very severe form of MFS, resulting in death from cardiac failure at the age of 4 months. Both of her parents were patients with MFS, each carrying a different FBN1 mutation. An authentic case of homozygosity in the FBN1 gene was later described in 2007 by de Vries et al in a Turkish consanguineous family. ${ }^{7}$ Subsequently, three other homozygous cases and two other compound heterozygous cases were reported. Since the original report in 1994, only seven cases of homozygous or compound heterozygous FBN1 mutations are found in the literature. All of them present severe clinical features.

Homozygous mutations in inherited autosomaldominant disorder are rare events. Here we have reported the experience of our team which performed systematic screening of the FBN1 gene in patients suspected for MFS. The laboratory has performed diagnostic FBN1 gene sequencing since 1998. More than 2500 probands have been tested for molecular diagnosis and 1400 heterozygous mutational events were found. Herein we have reported the surprising finding that nine probands carried homozygous or compound heterozygous mutations. From these molecular findings, complete clinical features were gathered in these nine families and were compared with what is described in the literature. In contrast to previous reports, this study 
reports on the finding of these events in a general patient population and not in selected severe forms of MFS alone.

\section{METHODS}

\section{Patients}

This study included all the patients followed by the 'Centre National Maladies Rares-Syndrome de Marfan et apparentés', the French National Reference centre located at Bichat Hospital (coordinator Professor Guillaume Jondeau) and by the seven affiliated 'Competence centres' located throughout the country.

Clinical diagnosis was established according to the revised Ghent nosology. ${ }^{1}$ Patients were examined by several physicians: cardiologists, ophthalmologists, geneticists, rheumatologists or paediatricians (depending on their age) with specific evaluation of clinical features included in MFS. Systematic slit-lamp examination, cardiac ultrasonography and radiological investigations were also performed. Dural ectasia was looked for by imaging. Systemic score was calculated as described in the revised Ghent nosology. ${ }^{1}$

Between 1998 and 2015, blood samples were obtained for 2500 consecutive unselected probands referred nationwide to our laboratory for molecular diagnosis because of suspected MFS, whatever the severity. Informed consent was provided for all patients in agreement with the requirements of French bioethics laws. Referral criteria for molecular testing of FBN1 remained unchanged since 1998 and were the following: (1) patients must present features in at least two systems and one major feature as described in the first Ghent nosology ${ }^{8}$ and (2) an exception is made for patients with familial autosomal dominant or sporadic isolated ectopia lentis.

\section{DNA amplification and mutation detection}

Genomic DNA was isolated from peripheral blood leucocytes by standard procedures already reported. ${ }^{9}$ The FBN1 gene has been systematically screened in patients suspected of MFS by bidirectional sequencing of all coding exons and closely flanking intronic sequences in each patient (Big Dye terminators kit, ABI 3100 Genetic Analyzer, Applied Biosystems, Warrington, Cheshire, UK; primers are available on request). Since 2014, the FBN1 gene has been screened by Next-Generation Sequencing using MARFAN MASTR Assay (Multiplicom) on MiSeq (Illumina), with bioinformatics analysis of FASTQ files (CLC Genomics Workbench (CLCBio)). Once a mutation is found in this way, it is systematically confirmed by bidirectional Sanger sequencing of the altered exon. When the mutation alters the regional restriction map, the presence of the mutation is also checked by $\mathrm{PCR} /$ digestion using the appropriate restriction enzyme. In the case of a homozygous molecular event, it is ascertained with a minimum of two different primer pairs (primers available on request) and if possible on two different samples. When possible, familial segregation of mutations was investigated. Description of sequence variants is performed according to Human Genome Variation Society nomenclature. ${ }^{10}$ In brief, cDNA numbering with +1 corresponds to the $\mathrm{A}$ of ATG, the translation initiation codon in the reference sequence (FBN1: NM_000138). The initiation codon is codon 1. Exons numbering is historically made considering that exon 1 carries the initiation codon.

\section{Molecular aspects of variants}

Four traditional bioinformatics programs (Grantham score, Polyphen-2, ${ }^{11}$ SIFT $^{12}$ via Alamut Visual 2.7 (Interactive Biosoftware, Rouen, France) and UMD-Predictor ${ }^{13}$ ) were used to predict pathogenicity of missense variants. Effect on splicing was analysed through different tools including Human Splicing Finder, ${ }^{14}$ splicing prediction algorithm NNSPLICE $^{15}$ and MaxEntScan method. ${ }^{16}$ The locus-specific database UMD$\mathrm{FBN}^{3}$ was consulted for each identified variant. The existence of each molecular event was looked for in the Exome Aggregation Consortium (ExAC) database.

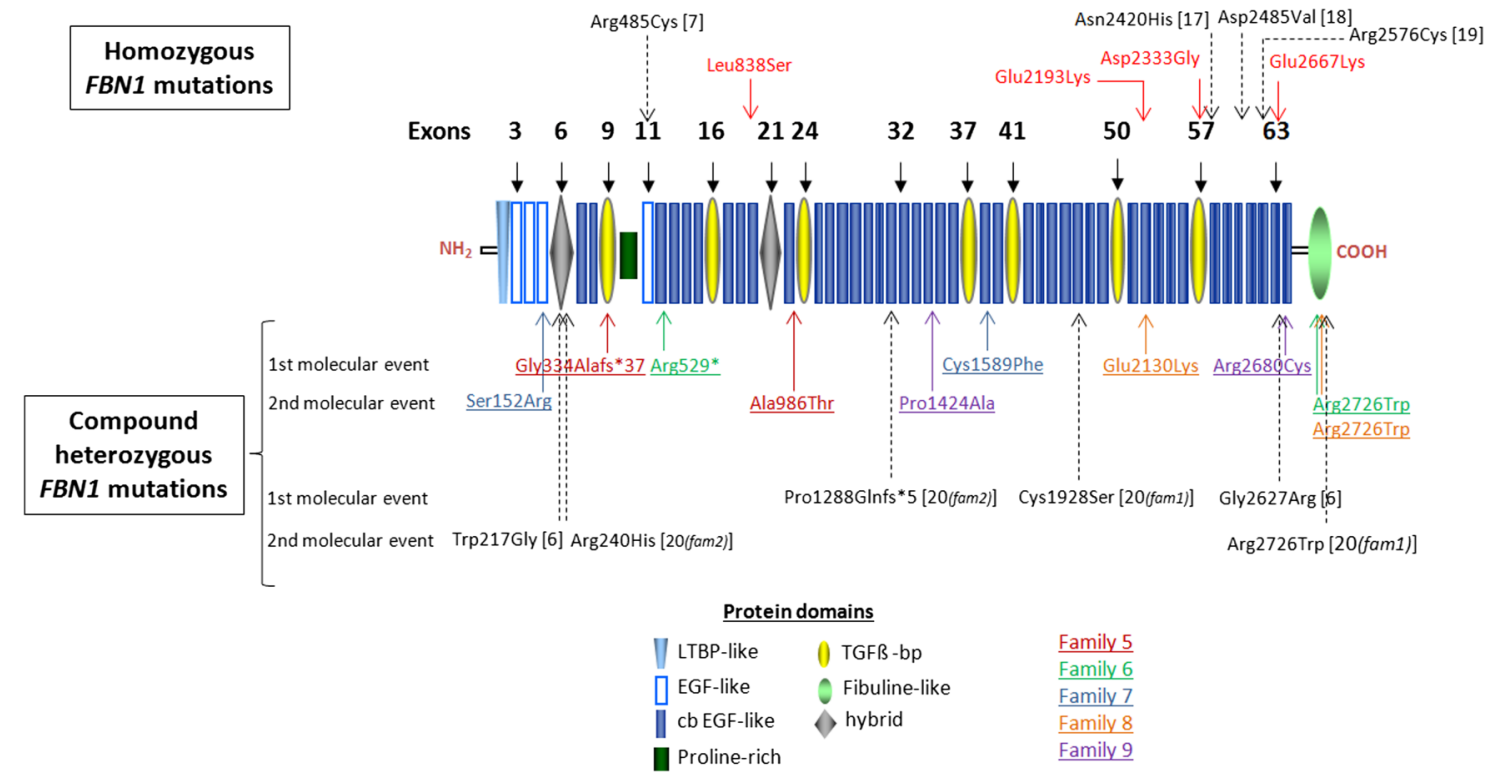

Figure 1 Representation of the localisation of homozygous and compound heterozygous mutations in fibrillin-1 protein. Exons encoding the different domains are numbered in bold. Homozygous mutations are represented above the figure (mutations reported here are in red). Compound heterozygous mutations are represented below the figure (mutations reported here are underlined and are colour-coded). Dashed arrows in black are used for previously reported mutations. Molecular events clearly in line with those usually observed in patients with Marfan syndrome (MFS) are named '1st molecular event'. cb-EGF, calcium-binding epidermal growth factor; EGF, epidermal growth factor; FBN1, gene encoding fibrillin-1; LTBP, latent TGF- $\beta$ binding protein; TGF $\beta$, transforming growth factor $\beta$. 


\section{RESULTS}

\section{Molecular aspects}

In the context of molecular screening of the FBN1 gene in patients with confirmed or potential diagnosis of heterozygous MFS or related allelic diseases, 2500 probands have been tested and 1400 heterozygous mutational events were found. Four probands presented homozygosity and 22 presented potential compound heterozygosity. Family investigations were possible in 12/ 22 cases and were performed to determine the respective locations of the events (in cis or trans). Five probands were shown to be compound heterozygotes. Thus, nine individuals carrying two mutated FBN1 alleles were identified, representing 14 molecular events (figure 1, table 1). Four probands carried missense homozygous FBN1 mutations: two were absent from both the ExAC and the UMD-FBN1 databases (p.Leu838Ser and p.Asp2333Gly) and two were reported in heterozygotes with an overall frequency of below 5/120000 in ExAC (p.Glu2667Lys and p.Glu2193Lys (the last was also found in the UMD-FBN1 database)). Three of them (p.Leu838Ser, p.Glu2193Lys and p.Glu2667Lys) affect highly conserved residues located in calcium-binding epidermal growth factor-like (cb-EGF) domains \#9, \#33 and \#63, respectively (figure 1). The forth mutation p.Asp2333Gly is located in transforming growth factor $\beta$-binding protein \#7 domain. It affects a highly conserved position which is the first nucleotide of exon 57; therefore, it may also affect splicing. Prediction tools

Table 1 Predictions of pathogenicity of homozygous and compound heterozygous gene encoding fibrillin-1 (FBN1) mutations in the report and in the literature

\begin{tabular}{|c|c|c|c|c|c|c|c|}
\hline Family no./reference & FBN1 mutations & $\begin{array}{l}\text { Grantham } \\
\text { distance } \\
(0-215)\end{array}$ & $\begin{array}{l}\text { Polyphen } \\
\text { human variant } \\
(0-1)\end{array}$ & SIFT (1-0) & $\begin{array}{l}\text { UMD-predictor } \\
(0-100)\end{array}$ & $\begin{array}{l}\text { Allele } \\
\text { frequency } \\
\text { in ExAC }\end{array}$ & $\begin{array}{l}\text { No. of probands } \\
\text { in UMD-FBN1 } \\
\text { database* }\end{array}$ \\
\hline \multicolumn{8}{|c|}{ Homozygous FBN1 mutations from the present report } \\
\hline Family 1 & c.2513T>C p.Leu838Ser & L (145) & $D(0.992)$ & Del (0) & Path (96) & 0 & 0 \\
\hline Family 2 & c.6577G >A p.Glu2193Lys & $S(56)$ & B (0.146) & $\mathrm{T}(0.35)$ & PM (63) & $3 / 121248$ & 1 \\
\hline Family 3 & c.6998A>G p.Asp2333Gly & M (94) & B $(0.433)$ & $\operatorname{Del}(0)$ & Path (90) & 0 & 0 \\
\hline Family 4 & c.7999G >A p.Glu2667Lys & $S(56)$ & B (0.183) & Del (0.03) & Path (75) & $4 / 121340$ & 0 \\
\hline \multicolumn{8}{|c|}{ Homozygous FBN1 mutations from the literature } \\
\hline de Vries et $a l^{7}$ & c. 1453 C $>$ T p.Arg485Cys & L (180) & $P(0.719)$ & Del (0) & Path (96) & 0 & 2 \\
\hline Khan et al ${ }^{17}$ & c. $7258 \mathrm{~A}>\mathrm{C}$ p.Asn2420His & $S(68)$ & $D(0.972)$ & Del (0) & Path (78) & 0 & 0 \\
\hline Hilhorst-Hofstee et al/ ${ }^{18}$ & c.7454A >T p.Asp2485Val & L (152) & $P(0.787)$ & Del $(0)$ & Path (93) & $5 / 120870$ & 0 \\
\hline Hogue et $a l^{19}$ & c.7726C $>$ T p.Arg2576Cys & L (180) & D (0.998) & Del $(0)$ & Path (96) & $1 / 120864$ & 2 \\
\hline \multicolumn{8}{|c|}{ Compound heterozygous FBN1 mutations from the present report } \\
\hline \multicolumn{8}{|l|}{ Family 5} \\
\hline First molecular event & $\begin{array}{l}\text { c.1001_1073del } \\
\text { p.Gly334Alafs*37 }\end{array}$ & - & - & - & - & 0 & 0 \\
\hline Second molecular event & c.2956G >A p.Ala986Thr & $S(58)$ & B $(0.190)$ & $\mathrm{T}(0.18)$ & Path (90) & $183 / 121370$ & 0 \\
\hline \multicolumn{8}{|l|}{ Family 6} \\
\hline First molecular event & c. 1585 C >T p.Arg529* & - & - & - & - & 0 & 7 \\
\hline Second molecular event & c.8176C >T p.Arg2726Trp & M (101) & B $(0.001)$ & Del $(0.01)$ & P Path (68) & $89 / 121410$ & 15 \\
\hline \multicolumn{8}{|l|}{ Family 7} \\
\hline First molecular event & c.4766G >T p.Cys1589Phe & L (205) & $D(0.994)$ & Del $(0)$ & Path (100) & 0 & 1 \\
\hline Second molecular event & c. $456 \mathrm{~T}>$ A p.Ser152Arg & M (110) & B $(0.040)$ & $\mathrm{T}(0.21)$ & Path (99) & 0 & 0 \\
\hline \multicolumn{8}{|l|}{ Family 8} \\
\hline First molecular event & c.6388G>A p.Glu2130Lys & $S(56)$ & $D(0.993)$ & $\operatorname{Del}(0)$ & P Path (72) & 0 & 6 \\
\hline Second molecular event & c.8176C >T p.Arg2726Trp & M (101) & $B(0.001)$ & Del $(0.01)$ & P Path (68) & $89 / 121410$ & 15 \\
\hline \multicolumn{8}{|l|}{ Family 9} \\
\hline First molecular event & c. $8038 \mathrm{C}>\mathrm{T}$ p.Arg2680Cys & L (180) & $P(0.873)$ & $\operatorname{Del}(0)$ & Path (96) & 0 & 4 \\
\hline Second molecular event & c. 4270 C >G p.Pro1424Ala & S (27) & $P(0.578)$ & $\mathrm{T}(0.07)$ & Path (90) & $22 / 121398$ & 10 \\
\hline \multicolumn{8}{|c|}{ Compound heterozygous FBN1 mutations from the literature } \\
\hline \multicolumn{8}{|l|}{ Karttunen et $a l^{6}$} \\
\hline First molecular event & c.7879G >A p.Gly2627Arg & M (125) & $D(1.000)$ & Del $(0)$ & Path (100) & 0 & 1 \\
\hline Second molecular event & c.649T>G p.Trp217Gly & L (184) & $D(1.000)$ & $\operatorname{Del}(0)$ & Path (96) & $1 / 121278$ & 0 \\
\hline \multicolumn{8}{|l|}{$\begin{array}{l}\text { Van Dijk et } a l^{20} \\
\text { Family } 1\end{array}$} \\
\hline First molecular event & c.5783G >C p.Cys1928Ser & M (112) & $P(0.492)$ & $\operatorname{Del}(0)$ & Path (100) & 0 & 1 \\
\hline Second molecular event & c.8176C >T p.Arg2726Trp & M (101) & B $(0.001)$ & Del (0) & P Path (68) & $89 / 121410$ & 15 \\
\hline \multicolumn{8}{|l|}{$\begin{array}{l}\text { Van Dijk et } a l^{20} \\
\text { Family } 2\end{array}$} \\
\hline First molecular event & $\begin{array}{l}\text { c. } 3861 \text { del } \\
\text { p.Pro1288Glnfs }{ }^{*}\end{array}$ & - & - & - & - & 0 & 1 \\
\hline Second molecular event & c. $719 \mathrm{G}>\mathrm{A}$ p.Arg240His & S (29) & $\mathrm{D}(0.987)$ & Del (0) & Path (78) & $1 / 120934$ & 0 \\
\hline
\end{tabular}

*Number of probands in the database out of 3146 probands, excluding the present case.

Grantham distance: L, large; M, moderate; S, small. Polyphen ${ }^{11}: B$, benign; D, probably damaging; P, possibly damaging. SIFT ${ }^{12}$ : Del, deleterious; T, tolerated. UMD-predictor ${ }^{13}$ : Path, pathogenic; P Path, probably pathogenic; PM, probable polymorphism. Prediction score is indicated between brackets. UMD-FBN1 database was consulted in April 2016. 
were mainly in favour of the pathogenicity of these mutations (table 1). When possible, the study of family members showed good segregation with the disease (figure 2, table 2). Four homozygous FBN1 mutations are described in the literature. $^{717-19}$ Three of them are also located in cb-EGF domains (\#37, \#39 and 41) and one is in EGF-like domain \#4 (figure $1)$. In two instances, the mutation introduces a new cysteine residue, $^{719}$ a mutation known to affect proper disulfide bond formation and disrupts domain conformation. Taken together, the eight homozygous mutations (this report and the literature) are all missense and are potentially associated with a dominant negative effect. Moreover, no homozygous mutation was found in exons 24-32, the hotspot of mutations found in cases of neonatal MFS. ${ }^{4}$ Finally, $5 / 8$ homozygous mutations strikingly cluster at the $3^{\prime}$ end of the FBN1 gene (between exons 57 and 63) (figure 1).

In the five compound heterozygous probands, we arbitrarily named 'first molecular event' the mutations that are comparable with those most often seen in patients with MFS: premature termination codon (PTC) mutations, loss or gain of cysteine mutations or mutations affecting a highly conserved residue in a given domain. The other mutation observed in a given proband was thus named 'second molecular event'. The mutations found in the five compound heterozygotes from this study are listed in table 1 and figure 1 . Among the 'second molecular events' identified, p.Ser152Arg is absent from both the ExAC and the UMD-FBN1 databases. The mutation is located in EGF\#3 and affects a highly conserved residue. Three other variants were found in four patients (p.Ala986Thr, p.Pro1424Ala and p.Arg2726Trp) and are reported in the ExAC database. However their frequency is globally low and algorithms are generally in favour of pathogenicity. Furthermore, two have already been reported in UMD-FBN1 database.
Interestingly, none of the homozygous or compound heterozygous carriers from the present report and from the literature carried two PTC molecular events in the FBN1 gene.

\section{Clinical aspects}

Three out of four homozygous carriers (from families 2, 3 and 4) were adults who displayed the classical MFS features usually found in heterozygous carriers (table 2). The fourth homozygous subject (family 1) was discovered through systematic clinical and molecular family screening for MFS. At discovery, he was aged 8 years, had a low systemic score and no cardiovascular or ocular feature. He is the nephew of the family proband (subject III-1, figure 2) in whom the diagnosis of MFS was made. The existence of a consanguineous mating was documented in families 1, 3 and 4. These three families were of Algerian origin. Family 2 originated from the eastern part of France, no consanguinity was known and history taking revealed no other case of MFS or related disease. In this family, the proband's parents were not available for investigation.

In two families, heterozygous carriers of the family mutation were available for investigation: five in family 1 and three in family 4 (table 2 and figure 2). A diagnosis of MFS was made in $3 / 5$ from the first family and $2 / 3$ in the second family. The remaining carriers in each family were women who displayed only mild skeletal features. The same clinical observation was made in the parents of the proband in family 3. This would be in line with an autosomal recessive form of MFS as that reported by de Vries et al. ${ }^{7}$

All the five compound heterozygous subjects presented the classical MFS features found in heterozygous carriers (table 2). Relatives heterozygous for one of the two mutations were only available in two families. In family 5 (figure 3), two of the
Figure 2 Representation of pedigrees of the homozygous carriers from the present report. Full square/ circle=homozygous carrier. Half-full square/circle=heterozygous carrier. Empty square/circle=data not available. Arrow=proband.

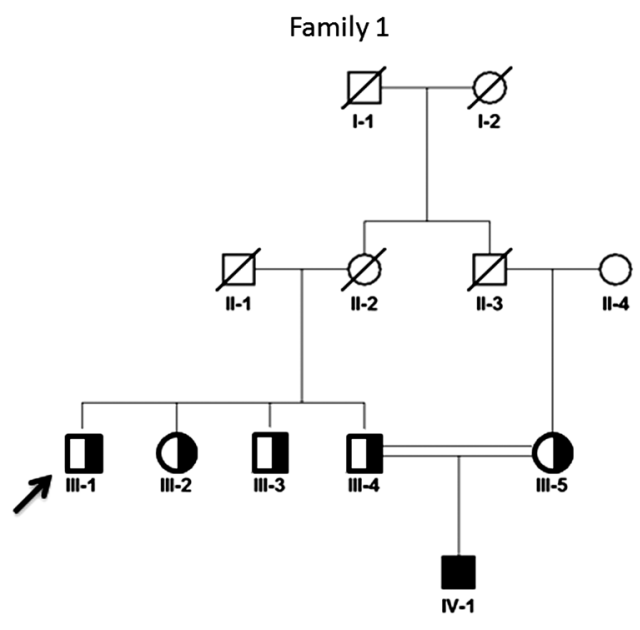

Family 2

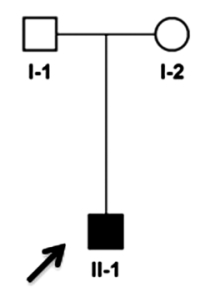

Family 3

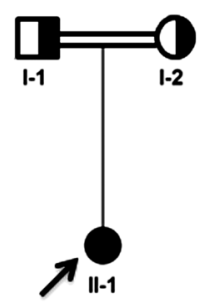

Family 4

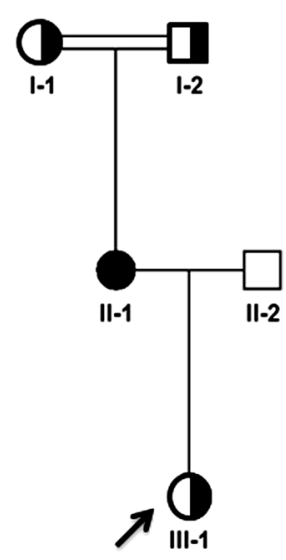


Table 2 Clinical features of homozygous and compound heterozygous gene encoding fibrillin-1 (FBN1) mutation carriers and their family members in the report and in the literature

\begin{tabular}{|c|c|c|c|c|c|c|c|c|c|}
\hline Family no. & $\begin{array}{l}\text { Individual } \\
\text { no. }\end{array}$ & Sex & FBN1 mutations & $\begin{array}{l}\text { Age at } \\
\text { diagnosis }\end{array}$ & $\begin{array}{l}\text { Age at the } \\
\text { last physical } \\
\text { examination }\end{array}$ & $\begin{array}{l}\text { Cardiovascular } \\
\text { system } \\
\text { (age at surgery) }\end{array}$ & $\begin{array}{l}\text { Ocular } \\
\text { system (age } \\
\text { at surgery) }\end{array}$ & $\begin{array}{l}\text { Systemic } \\
\text { score } \\
\text { (Ghent 2) }\end{array}$ & $\begin{array}{l}\text { Geographical } \\
\text { origin }\end{array}$ \\
\hline \multicolumn{10}{|c|}{ Homozygous vs heterozygous carriers from the present report } \\
\hline Family 1 & $\begin{array}{l}\text { IV-1 } \\
\text { III-1* } \\
\text { III-2 } \\
\text { III-3 } \\
\text { III-4 } \\
\text { III-5 }\end{array}$ & $\begin{array}{l}\text { Male } \\
\text { Male } \\
\text { Female } \\
\text { Male } \\
\text { Male } \\
\text { Female }\end{array}$ & $\begin{array}{l}\text { Leu838Ser (homozygous) } \\
\text { Leu838Ser } \\
\text { Leu838Ser } \\
\text { Leu838Ser } \\
\text { Leu838Ser } \\
\text { Leu838Ser }\end{array}$ & $\begin{array}{l}8 \\
63 \\
47 \\
48 \\
40 \\
39\end{array}$ & $\begin{array}{l}8 \\
63 \\
56 \\
48 \\
40 \\
39\end{array}$ & $\begin{array}{l}0 \\
\text { ao dil } \\
0 \\
\text { ao dil } \\
\text { ao diss (34) } \\
0\end{array}$ & $\begin{array}{l}0 \\
0 \\
0 \\
0 \\
0 \\
0\end{array}$ & $\begin{array}{l}3 \\
9 \\
8 \\
4 \\
3 \\
4\end{array}$ & Algeria \\
\hline Family 2 & $\mathrm{II}-1^{*}$ & Male & Glu2193Lys (homozygous) & 32 & 34 & ao dil & EL & 2 & France \\
\hline Family 3 & $\begin{array}{l}\mathrm{II}-1^{*} \\
\mathrm{I}-1 \\
\mathrm{I}-2\end{array}$ & $\begin{array}{l}\text { Female } \\
\text { Male } \\
\text { Female }\end{array}$ & $\begin{array}{l}\text { Asp2333Gly (homozygous) } \\
\text { Asp2333Gly } \\
\text { Asp2333Gly }\end{array}$ & $\begin{array}{l}26 \\
66 \\
59\end{array}$ & $\begin{array}{l}28 \\
66 \\
59\end{array}$ & $\begin{array}{l}\text { ao dil } \\
0 \\
0\end{array}$ & $\begin{array}{l}\text { EL (4) } \\
0 \\
0\end{array}$ & $\begin{array}{l}14 \\
7 \\
5\end{array}$ & Algeria \\
\hline Family 4 & $\begin{array}{l}\mathrm{II}-1 \\
\mathrm{I}-1 \\
\mathrm{I}-2 \\
\mathrm{III}-1^{*}\end{array}$ & $\begin{array}{l}\text { Female } \\
\text { Female } \\
\text { Male } \\
\text { Female }\end{array}$ & $\begin{array}{l}\text { Glu2667Lys (homozygous) } \\
\text { Glu2667Lys } \\
\text { Glu2667Lys } \\
\text { Glu2667Lys }\end{array}$ & $\begin{array}{l}51 \\
76 \\
78 \\
12\end{array}$ & $\begin{array}{l}53 \\
76 \\
79 \\
15\end{array}$ & $\begin{array}{l}\text { ao dil } \\
0 \\
\text { ao dil } \\
\text { ao dil }\end{array}$ & $\begin{array}{l}0 \\
\text { NA } \\
\text { NA } \\
0\end{array}$ & $\begin{array}{l}11 \\
2 \\
0 \\
7\end{array}$ & Algeria \\
\hline
\end{tabular}

\section{Homozygous vs heterozygous carriers from the literature}

\begin{tabular}{|c|c|c|c|c|c|c|c|c|c|}
\hline \multirow[t]{6}{*}{ de Vries et $a l^{7}$} & III-1* & Male & Arg485Cys (homozygous) & 22 & NA & ao diss & EL & 5 & \multirow[t]{6}{*}{ Turkey } \\
\hline & III-4 & Female & Arg485Cys (homozygous) & 13 & NA & 0 & EL & 3 & \\
\hline & II-1 & Male & Arg485Cys & NA & NA & 0 & 0 & 0 & \\
\hline & $\|-2$ & Female & Arg485Cys & NA & NA & 0 & 0 & 0 & \\
\hline & II-3 & Female & Arg485Cys & NA & NA & MVP & 0 & 2 & \\
\hline & II-4 & Male & Arg485Cys & NA & NA & 0 & 0 & 0 & \\
\hline \multirow[t]{3}{*}{ Khan et $a l^{17}$} & $\| 1-1$ * & Female & Asn2420His (homozygous) & 3 & NA & NA & EL & $\begin{array}{l}\text { NA (at } \\
\text { least 1) }\end{array}$ & \multirow[t]{3}{*}{ Saudi Arabia } \\
\hline & $\mathrm{I}-1$ & Male & Asn2420His & NA & NA & NA & 0 & NA & \\
\hline & $1-2$ & Female & Asn2420His & NA & NA & NA & 0 & NA & \\
\hline \multirow{16}{*}{$\begin{array}{l}\text { Hilhorst- } \\
\text { Hofstee } \\
\text { et al }\end{array}$} & II-15 & Male & Asp2485Val (homozygous) & NA & 9 & ao dil & EL & 6 & \multirow[t]{16}{*}{ Turkey } \\
\hline & III-1* & Female & Asp2485Val (homozygous) & NA & 22 & ao dil & EL & 7 & \\
\hline & III-3 & Male & Asp2485Val (homozygous) & NA & 10 & ao dil & EL & 5 & \\
\hline & I-1 & Male & Asp2485Val & NA & 61 & 0 & 0 & 0 & \\
\hline & $1-5$ & Male & Asp2485Val & NA & 57 & 0 & 0 & 0 & \\
\hline & $1-6$ & Female & Asp2485Val & NA & 56 & 0 & 0 & 0 & \\
\hline & II-1 & Male & Asp2485Val & NA & 44 & ao dil & 0 & 1 & \\
\hline & II-2 & Male & Asp2485Val & NA & 41 & 0 & 0 & 0 & \\
\hline & II-3 & Female & Asp2485Val & NA & 37 & 0 & 0 & 1 & \\
\hline & II-4 & Male & Asp2485Val & NA & 35 & 0 & 0 & 1 & \\
\hline & II-5 & Female & Asp2485Val & NA & 33 & 0 & 0 & 0 & \\
\hline & II-6 & Male & Asp2485Val & NA & 42 & 0 & 0 & 0 & \\
\hline & II-7 & Female & Asp2485Val & NA & 43 & 0 & 0 & 6 & \\
\hline & II-8 & Female & Asp2485Val & NA & 55 & 0 & 0 & 2 & \\
\hline & II-9 & Female & Asp2485Val & NA & 48 & 0 & 0 & 1 & \\
\hline & II-12 & Female & Asp2485Val & NA & 37 & 0 & 0 & 0 & \\
\hline \multirow[t]{4}{*}{ Hogue et $a l^{19}$} & $\| 1$-1* & Female & Arg2576Cys (homozygous) & 20 & NA & ao diss (20) & EL & 11 & \multirow[t]{4}{*}{ Mexico } \\
\hline & $\mathrm{I}-1$ & Male & Arg2576Cys & NA & NA & NA & 0 & 0 & \\
\hline & $1-2$ & Female & Arg2576Cys & NA & NA & NA & 0 & 0 & \\
\hline & III-1 & Male & Arg2576Cys & $\mathrm{NA}$ & NA & 0 & 0 & 1 & \\
\hline
\end{tabular}

Compound heterozygous vs heterozygous carriers from the present report

\begin{tabular}{|c|c|c|c|c|c|c|c|c|}
\hline \multirow[t]{4}{*}{ Family 5} & $1-2 *$ & Male & Gly334Alafs*37/Ala986Thr & 25 & 29 & ao diss (25) & flat corneas & 6 \\
\hline & II-2 & Male & Gly334Alafs*37 & 6 & 6 & ao dil & EL & 2 \\
\hline & II-3 & Male & Gly334Alafs*37 & 3 & 3 & ao dil & NA & 5 \\
\hline & $\|-1$ & Male & Ala986Thr & 8 & 10 & ao dil & EL & 0 \\
\hline \multirow[t]{7}{*}{ Family 6} & III-3* & Male & Arg529*/Arg2726Trp & 8 & 18 & ao dil & EL & 6 \\
\hline & II-1 & Male & Arg529* & 30 & 51 & ao diss (46) & EL & 5 \\
\hline & $\mathrm{I}-1$ & Male & Arg529* mosaic & 76 & 76 & 0 & 0 & 2 \\
\hline & $\|-2$ & Female & Arg2726Trp & 45 & 45 & 0 & 0 & 4 \\
\hline & II-3 & Male & Arg2726Trp & 43 & 43 & 0 & flat corneas & 2 \\
\hline & III-1 & Male & Arg2726Trp & 5 & 22 & 0 & 0 & 1 \\
\hline & III-2 & Male & Arg2726Trp & 3 & 19 & 0 & 0 & 5 \\
\hline \multirow[t]{2}{*}{ Family 7} & $\mathrm{II}-2^{*}$ & Female & Cys1589Phe/Ser152Arg & 53 & 55 & $\begin{array}{l}\text { ao dil (Bentall } \\
\text { at } 51 \text { ) }\end{array}$ & EL (40) & 7 \\
\hline & III-1 & Female & Cys1589Phe & 26 & 28 & ao dil & EL (20) & 3 \\
\hline
\end{tabular}


Table 2 Continued

\begin{tabular}{|c|c|c|c|c|c|c|c|c|c|}
\hline Family no. & $\begin{array}{l}\text { Individual } \\
\text { no. }\end{array}$ & Sex & FBN1 mutations & $\begin{array}{l}\text { Age at } \\
\text { diagnosis }\end{array}$ & $\begin{array}{l}\text { Age at the } \\
\text { last physical } \\
\text { examination }\end{array}$ & $\begin{array}{l}\text { Cardiovascular } \\
\text { system } \\
\text { (age at surgery) }\end{array}$ & $\begin{array}{l}\text { Ocular } \\
\text { system (age } \\
\text { at surgery) }\end{array}$ & $\begin{array}{l}\text { Systemic } \\
\text { score } \\
\text { (Ghent 2) }\end{array}$ & $\begin{array}{l}\text { Geographical } \\
\text { origin }\end{array}$ \\
\hline \multirow[t]{3}{*}{ Family 8} & $\mathrm{II}-2^{*}$ & Female & Glu2130Lys/Arg2726Trp & 41 & 49 & $\begin{array}{l}\text { ao dil (Bentall } \\
\text { at 29) }\end{array}$ & EL & 10 & \\
\hline & $\mathrm{I}-2$ & Female & Arg2726Trp & 67 & 67 & 0 & 0 & 1 & \\
\hline & III-1 & Male & Arg2726Trp & 16 & 16 & 0 & 0 & 1 & \\
\hline \multirow[t]{2}{*}{ Family 9} & $\mathrm{I}-2^{*}$ & Female & Arg2680Cys/Pro1424Ala & 30 & 30 & ao dil & myopia & 8 & \\
\hline & II-1 & Male & Arg2680Cys & 2 & 2 & NA & EL & 0 & \\
\hline
\end{tabular}

Compound heterozygous vs heterozygous carriers from the literature

\begin{tabular}{|c|c|c|c|c|c|c|c|c|}
\hline $\begin{array}{l}\text { Karttunen } \\
\text { et } a l^{6}\end{array}$ & $\begin{array}{l}\mathrm{II}-1 \text { * } \\
\mathrm{I}-2 \\
\mathrm{I}-1\end{array}$ & $\begin{array}{l}\text { Male } \\
\text { Female } \\
\text { Male }\end{array}$ & $\begin{array}{l}\text { Gly2627Arg/Trp217Gly } \\
\text { Gly2627Arg } \\
\text { Trp217Gly }\end{array}$ & $\begin{array}{l}1 \text { week } \\
\text { teenager } \\
\text { NA }\end{array}$ & $\begin{array}{l}3 \text { months } \\
33 \\
35\end{array}$ & $\begin{array}{l}\text { NA } \\
0 \\
\text { ao dil }\end{array}$ & $\begin{array}{l}\text { NA } \\
\text { myopia } \\
\text { EL }\end{array}$ & $\begin{array}{l}4 \\
5 \\
6\end{array}$ \\
\hline $\begin{array}{l}\text { Van Dijk } \\
\text { et } a l^{20}\end{array}$ & II-1* & Male & Cys1928Ser/Arg2726Trp & 12 & 20 & $\begin{array}{l}\text { ao dil (Bentall } \\
\text { at 19) }\end{array}$ & EL & 9 \\
\hline \multirow[t]{3}{*}{ Family 1} & $\mathrm{I}-2$ & Female & Cys1928Ser & 20 & NA & $\begin{array}{l}\text { ao dil (Bentall at } \\
35 \text { ) }\end{array}$ & EL & 7 \\
\hline & $\mathrm{I}-1$ & Male & Arg2726Trp & NA & 54 & 0 & 0 & 3 \\
\hline & $\|-2$ & Male & Arg2726Trp & NA & 17 & 0 & 0 & 4 \\
\hline $\begin{array}{l}\text { Van Dijk } \\
\text { et } a l^{20}\end{array}$ & II-1* & Female & Pro1288Glnfs*5/Arg240His & 5 & 19 & 0 & EL & 7 \\
\hline \multirow[t]{3}{*}{ Family 2} & II-2 & Male & Pro1288GInfs * $5 /$ Arg $240 \mathrm{His}$ & 5 & 15 & $\begin{array}{l}\text { ao dil (Bentall } \\
\text { at 15) }\end{array}$ & EL & 5 \\
\hline & $\mathrm{I}-2$ & Female & Pro1288Glnfs*5 & 31 & NA & 0 & EL & 5 \\
\hline & $\mathrm{I}-1$ & Male & Arg240His & NA & NA & 0 & 0 & 1 \\
\hline
\end{tabular}

Homozygous or compound heterozygous carriers are in bold.

*Proband in the family.

ao dil, aortic dilatation; ao diss, aortic dissection; EL, ectopia lentis; MVP, mitral valve prolapse; NA, not available.

proband's sons (II-2 and II-3) inherited p.Gly334Alafs*37 and both displayed a dilated aortic root at the age of 6 and 3 years, respectively. Conversely their brother carried the second paternal mutation (p.Ala986Thr). At the clinical level, he also displayed dilated aortic root at 8 years. In family 6 (figure 3), the proband (III-3) inherited p.Arg529* from his father (II-1) in whom diagnosis of MFS was made. Molecular screening in the grandfather (I-1) revealed a somatic (blood cells) mosaïcism and no clinical manifestation at 76 years. The second molecular event was inherited from the maternal family of the proband. Both the mother (II-2) and her brother (II-3) carried p. Arg2726Trp and displayed mild skeletal features. Interestingly, this mutation was also found in family 8 (compound heterozygosity) where it is present as a heterozygous event in two subjects (I-2 and III-1). Both also displayed mild skeletal features. Finally in the two remaining families (figure 3), heterozygous carriers of only one of the two family mutations were available for investigation: one p.Cys1589Phe carrier in family 7 with a definite diagnosis of MFS at age 26 years and one p.Arg2680Cys carrier in family 9 with ectopia lentis operated on at age 2 years (figure 3 ).

\section{DISCUSSION}

In the context of molecular diagnosis of MFS and related disorders, our laboratory has performed systematic sequencing of the entire FBN1 gene in over 2500 probands leading to the identification of over 1400 mutations. In the course of this systematic work, completed by familial screening, we identified 26 subjects carrying two molecular events in the FBN1 gene: homozygosity was observed in four, while two distinct events were found in the remaining 22. Through family investigation, compound heterozygosity was confirmed in five probands. These findings of homozygous and compound heterozygous subjects were unexpected since they were found in probands or subjects sampled through family investigation for classic MFS. Therefore, heterozygosity for molecular events in the FBN1 gene was expected. Overall, we identified four homozygous and five compound heterozygous subjects. This is a rare finding since it represents $0.64 \%$ of our series. It is also rare worldwide since the UMD-FBN1 database (the most comprehensive list of mutations) contains information of 3146 probands and only five are homozygotes and seven are compound heterozygotes. In the literature, only seven comparable cases (four homozygotes and three compound heterozygotes) have been reported (tables 1 and 2). In four reports, ${ }^{7}{ }^{17-19}$ seven homozygous individuals among four families were described. These were severe cases of MFS with an age at diagnosis ranging from 3 to 22 years. All of them presented with ectopia lentis, two of them had an ascending aortic dissection and one underwent preventive aortic surgery. In two other reports, ${ }^{6}{ }^{20}$ four compound heterozygous individuals among three families were described. These were also very severe MFS cases diagnosed between the first week of life and 12 years. In brief, these cases were identified either because of great clinical severity ${ }^{6} 19{ }^{20}$ recessive MFS $^{718}$ or consanguineous families. ${ }^{17}$ Therefore, they cannot be compared with the ones we report since our findings were in a wider molecular context but in a very different clinical setting, that is, diagnosis of classic form of MFS.

Taken together, our results and literature reports have identified eight cases of homozygous FBN1 mutations associated with MFS. In 7/8 instances, subjects belonged to pedigrees with consanguineous loops from geographical regions where inbreeding is well documented (Algeria (this report), Turkey, ${ }^{7}{ }^{18}$ Saudi Arabia, ${ }^{17}$ Mexico $^{19}$ (table 2)). The clinical presentations of published probands tend to affect all systems (notably ocular and cardiovascular) and appear at an early age. Conversely, the 
Figure 3 Representation of pedigrees of the compound heterozygous carriers from the present report. Half-dot square/circle=heterozygous carrier for the first molecular event. Half-hatched square/circle=heterozygous carrier for the second molecular event. Empty square/circle=data not available. Probable mosaïcism. Arrow=proband.
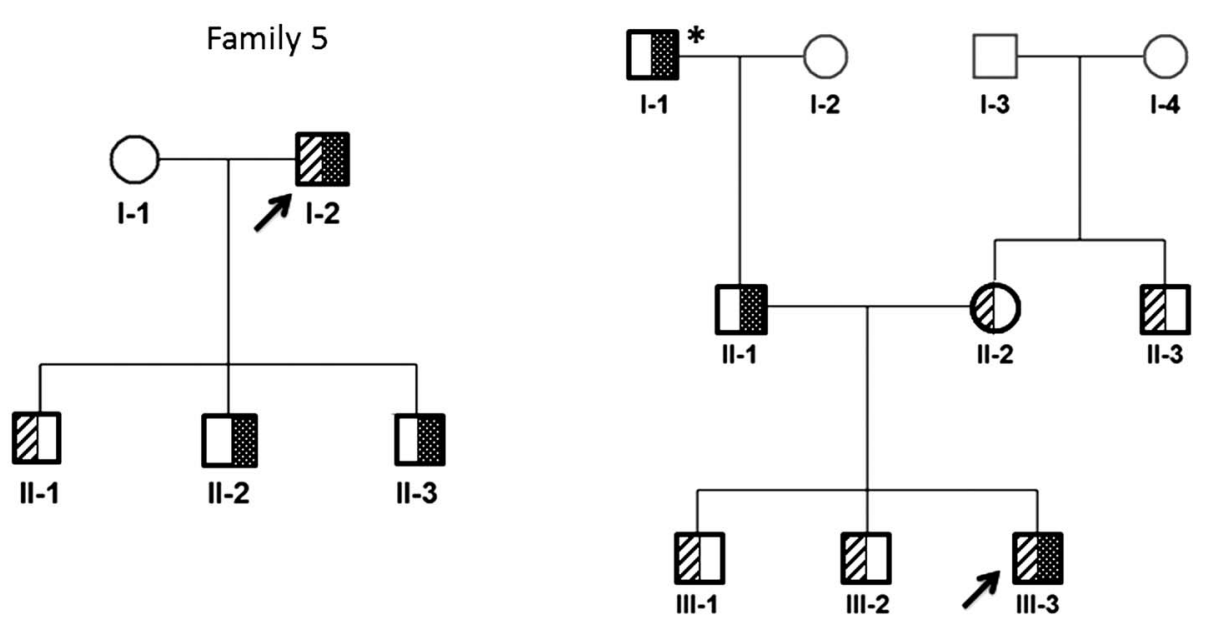

Family 7

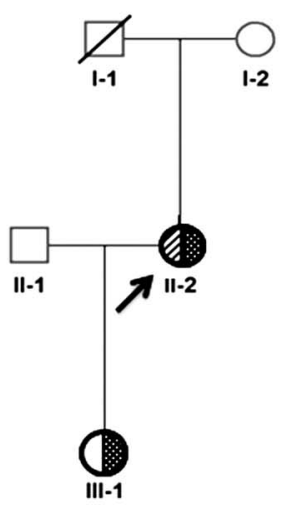

Family 8

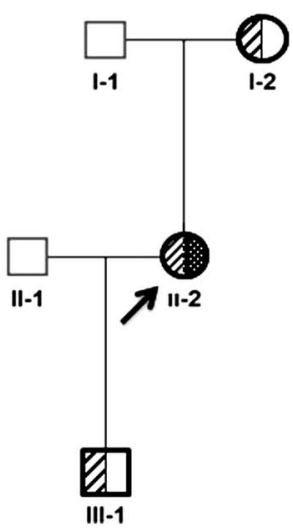

Family 6

Family 9

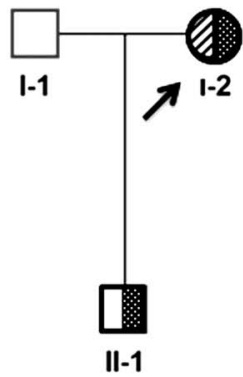

homozygous French patients have milder phenotypes, not different from phenotypes observed in the patients carrying only one mutation in the FBN1 gene. At the molecular level, the eight molecular events are all missense and strikingly cluster at the $3^{\prime}$ end of the FBN1 gene (5/8 between exons 57 and 63 (figure 1)). In this region (exons 59-63), Palz et $a l^{21}$ described a cluster of mutations associated with milder phenotypes with less aortic involvement. The mutations found in the French probands provide further support to this observation which could partly explain the lack of a severe MFS presentation in homozygous carriers. None of the homozygous or compound heterozygous cases carried two molecular events usually observed in patients with MFS, that is to say PTC mutations, loss or gain of cysteine mutations or mutations affecting a highly conserved residue in a given domain. Comparatively, in our cohort, approximately $30 \%$ of all mutations are PTC mutations and $35 \%$ are loss or gain of cysteine.

Regarding compound heterozygous FBN1 mutations associated with MFS, a total of nine cases have been identified combining our results and the literature. The clinical presentations of the three published probands are generally severe and appear at a much earlier age (1 week to 12 years) as compared with the French compound heterozygotes (8 to 53 years) and the homozygous probands described above. Interestingly, the p.Arg2726Trp mutation is found in three probands and is associated with p.Arg529* in family 6 (diagnosis at 8 years), with p.Glu2130Lys in family 8 (aortic surgery at 29 years) and with p. Cys1928Ser as reported in a 12-year-old proband by Van Dijk et $a .^{20}$ The pathogenicity of p.Arg2726Trp was demonstrated in vitro where it was shown to inhibit furin/PACE processing of profibrillin. $^{22}$ At the clinical level, the mutation was described in heterozygous patients with isolated skeletal features of MFS and/or high stature. ${ }^{22} 23$ Interestingly, cases of incomplete penetrance have been reported for heterozygous carriers of this mutation. ${ }^{23}$ This is in line with the heterozygous carriers of families 6 and 8 who display few clinical features as well as one reported by Van Dijk et $a l^{20}$ (table 2). Therefore, it can be speculated that $\mathrm{p}$. Arg2726Trp displays lack of penetrance to explain the lack of a severe phenotype in compound heterozygotes who carry this mutation. This conclusion could also apply to mutations p. Ala986Thr (family 5) and p.Pro1424Ala (family 9). Indeed, in our series as well as in the UMD-FBN1 database, heterozygote carriers are known for these two mutations and they display features in all systems, thus presenting an unequivocal diagnosis of MFS. Therefore, the lack of an aggravated phenotype in compound heterozygotes from families 5 and 9 is surprising and can only be explained this far through incomplete penetrance. Finally, the last proband (family 7) carries a mutation comparable with those usually found in patients (p.Cys1589Phe) and a second event has never been reported in any database and predicted to be 
deleterious (p.Ser152Arg). The lack of an aggravated disease in the proband again is in favour of an overall lack of penetrance due to an unexplained mechanism.

In conclusion, we report nine new patients with homozygous or compound heterozygous mutations in the FBN1 gene. This is the first report evaluating the frequency of occurrence of these events in the Marfan population in relation to mutation in the FBN1 gene $(0.64 \%)$, as it is the first report of these findings during systematic screening of the complete gene in this population. These patients complete the clinical data available for these rare molecular occurrences. Contrary to previous reports, these probands were not identified through early and very severe clinical presentations but unexpectedly in the course of molecular diagnosis of patients presenting classic form of MFS. Therefore, these rare events should not be overlooked, especially among consanguineous families. Finally, no prediction of disease severity should be extrapolated as evidenced by the lack of an aggravated phenotype in carriers.

\section{Web resources}

The URLs for data presented herein are as follows:

HGVS, Nomenclature for the description of sequence variants: http://www.hgvs.org/mutnomen/

ExAC database: http://exac.broadinstitute.org

PolyPhen-2: http://genetics.bwh.harvard.edu/pph2/

UMD-Predictor: http://umd-predictor.eu/

UMD-FBN1 mutations database: http://www.umd.be/FBN1/

\author{
Author affiliations \\ 'Département de Génétique et Centre de Référence Maladies Rares Syndrome de \\ Marfan et pathologies apparentées, Assistance Publique-Hôpitaux de Paris, Hôpital \\ Bichat, Paris, France \\ ${ }^{2}$ LVTS, INSERM U1148, Université Paris Diderot, Hôpital Bichat, Paris, France \\ ${ }^{3}$ LVTS, INSERM U1148, Hôpital Bichat, Paris, France \\ ${ }^{4}$ Hôpital de Brabois, Service de Génétique Clinique, Centre Hospitalier Universitaire \\ de Nancy, Vandoeuvre-lès-Nancy, France \\ ${ }^{5}$ Hôpital Femme-Mère-Enfant, Service de Génétique Clinique, Centre Hospitalier \\ Universitaire de Lyon, Bron, France \\ ${ }^{6}$ GH Pellegrin, Service de Génétique Médicale, Centre Hospitalier Universitaire de \\ Bordeaux, Bordeaux, France \\ ${ }^{7}$ Hôpital Bichat, Centre de Référence Maladies Rares, Syndrome de Marfan et \\ pathologies apparentéés, Assistance Publique-Hôpitaux de Paris, Paris, France \\ ${ }^{8}$ Hôpital Sud, Service de Génétique Clinique, Centre Hospitalier Universitaire de \\ Rennes, Rennes, France \\ ${ }^{9}$ Hôpital François Mitterrand, Centre de Génétique-Dijon, Centre Hospitalier \\ Universitaire Dijon, Dijon, France \\ ${ }^{10}$ Hôpital Timone Adultes, Service de Chirurgie vasculaire, Assistance Publique- \\ Hôpitaux de Marseille, Marseille, France \\ ${ }^{11}$ Centre Hospitalier Universitaire de Toulouse, Hôpital des Enfants, Service de \\ Cardiologie, Toulouse, France \\ ${ }^{12}$ Aix Marseille Univ, INSERM, GMGF, Marseille, France \\ ${ }^{13}$ Faculté de Médecine, Aix-Marseille Université, Marseille, France
}

Acknowledgements The authors wish to thank all colleagues who referred probands and families for molecular investigations. They also acknowledge the major contribution of all members of the 'Centre de Référence Maladies Rares Syndrome de Marfan et apparentés'.

Contributors BL, SD-G, SN, DL, OM, SO, LF, LB, TE, ML, MS, LG and GJ: patient referral, in-depth clinical data and family pedigrees. GCB: UMD-FBN1 database expertise. PA, NH, MA and CB: laboratory work, acquisition and interpretation of data. PA, LG, GJ and CB: study design, supervision, manuscript preparation and critical revision.

Funding This work was supported by 'Programme Hospitalier de Recherche Clinique' AOM10108 and CRC15014 (CB and GJ), 'Agence Nationale de la Recherche' NONAGES, ANR-14-CE15-0012-01 (CB and GJ), 'Fédération de Cardiologie' (GJ) and "Société de Cardiologie" (GJ).

Competing interests None declared.

Patient consent Obtained.

Ethics approval French Bioethics Laws.

\section{REFERENCES}

1 Loeys BL, Dietz HC, Braverman AC, Callewaert BL, De Backer J, Devereux RB, Hilhorst-Hofstee Y, Jondeau G, Faivre L, Milewicz DM, Pyeritz RE, Sponseller PD, Wordsworth P, De Paepe AM. The revised Ghent nosology for the Marfan syndrome. J Med Genet 2010;47:476-85.

2 Faivre L, Collod-Beroud G, Adès L, Arbustini E, Child A, Callewaert BL, Loeys B, Binquet C, Gautier E, Mayer K, Arslan-Kirchner M, Grasso M, Beroud C, Hamroun D, Bonithon-Kopp C, Plauchu H, Robinson PN, De Backer J, Coucke P, Francke U, Bouchot O, Wolf JE, Stheneur C, Hanna N, Detaint D, De Paepe A, Boileau C, Jondeau $\mathrm{G}$. The new Ghent criteria for Marfan syndrome: what do they change? Clin Genet 2012;81:433-42.

3 Collod-Béroud G, Le Bourdelles S, Ades L, Ala-Kokko L, Booms P, Boxer M, Child A, Comeglio P, De Paepe A, Hyland JC, Holman K, Kaitila I, Loeys B, Matyas G, Nuytinck L, Peltonen L, Rantamaki T, Robinson P, Steinmann B, Junien C, Béroud C, Boileau C. Update of the UMD-FBN1 mutation database and creation of an FBN1 polymorphism database. Hum Mutat 2003;22:199-208.

4 Faivre L, Collod-Beroud G, Loeys BL, Child A, Binquet C, Gautier E, Callewaert B, Arbustini E, Mayer K, Arslan-Kirchner M, Kiotsekoglou A, Comeglio P, Marziliano N, Dietz HC, Halliday D, Beroud C, Bonithon-Kopp C, Claustres M, Muti C, Plauchu H, Robinson PN, Adès $L C$, Biggin $A$, Benetts $B$, Brett $M$, Holman KJ, De Backer J, Coucke P, Francke U, De Paepe A, Jondeau G, Boileau C. Effect of mutation type and location on clinical outcome in 1,013 probands with Marfan syndrome or related phenotypes and FBN1 mutations: an international study. Am J Hum Genet 2007;81:454-66

5 Dietz HC, Cutting GR, Pyeritz RE, Maslen CL, Sakai LY, Corson GM, Puffenberger EG, Hamosh A, Nanthakumar EJ, Curristin SM. Marfan syndrome caused by a recurrent de novo missense mutation in the fibrillin gene. Nature 1991;352:337-9.

6 Karttunen L, Raghunath M, Lönnqvist L, Peltonen L. A compound-heterozygous Marfan patient: two defective fibrillin alleles result in a lethal phenotype. Am J Hum Genet 1994;55:1083-91.

7 de Vries BB, Pals G, Odink R, Hamel BC. Homozygosity for a FBN1 missense mutation: clinical and molecular evidence for recessive Marfan syndrome. Eur I Hum Genet 2007;15:930-5.

8 De Paepe A, Devereux RB, Dietz HC, Hennekam RC, Pyeritz RE. Revised diagnostic criteria for the Marfan syndrome. Am J Med Genet 1996;62:417-26.

9 Stheneur C, Collod-Béroud G, Faivre L, Buyck JF, Gouya L, Le Parc JM, Moura B, Muti C, Grandchamp B, Sultan G, Claustres M, Aegerter P, Chevallier B, Jondeau $G$, Boileau $C$. Identification of the minimal combination of clinical features in probands for efficient mutation detection in the FBN1 gene. Eur J Hum Genet 2009;17:1121-8.

10 den Dunnen JT, Antonarakis SE. Mutation nomenclature extensions and suggestions to describe complex mutations: a discussion. Hum Mutat 2000;15:7-12.

11 Adzhubei IA, Schmidt S, Peshkin L, Ramensky VE, Gerasimova A, Bork P, Kondrashov AS, Sunyaev SR. A method and server for predicting damaging missense mutations. Nat Methods 2010;7:248-9.

12 Kumar P, Henikoff S, Ng PC. Predicting the effects of coding non-synonymous variants on protein function using the SIFT algorithm. Nat Protoc 2009;4:1073-81.

13 Frédéric MY, Lalande M, Boileau C, Hamroun D, Claustres M, Béroud C, Collod-Béroud G. UMD-predictor, a new prediction tool for nucleotide substitution pathogenicity—application to four genes: FBN1, FBN2, TGFBR1, and TGFBR2. Hum Mutat 2009;30:952-9.

14 Desmet FO, Hamroun D, Lalande M, Collod-Béroud G, Claustres M, Béroud C. Human Splicing Finder: an online bioinformatics tool to predict splicing signals. Nucleic Acids Res 2009:37:e67.

15 Adams MD, Celniker SE, Holt RA, Evans CA, Gocayne JD, Amanatides PG, Scherer SE, Li PW, Hoskins RA, Galle RF, George RA, Lewis SE, Richards S, Ashburner M, Henderson SN, Sutton GG, Wortman JR, Yandell MD, Zhang Q, Chen LX, Brandon RC, Rogers YH, Blazej RG, Champe M, Pfeiffer BD, Wan KH, Doyle C, Baxter EG, Helt G, Nelson CR, Gabor GL, Abril JF, Agbayani A, An HJ, Andrews-Pfannkoch C, Baldwin D, Ballew RM, Basu A, Baxendale J, Bayraktaroglu L, Beasley EM, Beeson $\mathrm{KY}$, Benos PV, Berman BP, Bhandari D, Bolshakov S, Borkova D, Botchan MR, Bouck J, Brokstein P, Brottier P, Burtis KC, Busam DA, Butler H, Cadieu E, Center A, Chandra I, Cherry JM, Cawley S, Dahlke C, Davenport LB, Davies P, de Pablos B, Delcher A, Deng Z, Mays AD, Dew I, Dietz SM, Dodson K, Doup LE, Downes M, Dugan-Rocha S, Dunkov BC, Dunn P, Durbin KJ, Evangelista CC, Ferraz C, Ferriera S, Fleischmann W, Fosler C, Gabrielian AE, Garg NS, Gelbart WM, Glasser K, Glodek A, Gong F, Gorrell JH, Gu Z, Guan P, Harris M, Harris NL, Harvey D, Heiman TJ, Hernandez JR, Houck J, Hostin D, Houston KA, Howland TJ, Wei MH, Ibegwam C, Jalali M, Kalush F, Karpen GH, Ke Z, Kennison JA, Ketchum KA, Kimmel BE, Kodira CD, Kraft C, Kravitz S, Kulp D, Lai Z, Lasko P, Lei Y, Levitsky AA, Li J, Li Z, Liang $Y$, Lin $X$, Liu X, Mattei B, Mclntosh TC, McLeod MP, McPherson D, Merkulov G, Milshina NV, Mobarry C, Morris J, Moshrefi A, Mount SM, Moy M, Murphy B, Murphy L, Muzny DM, Nelson DL, Nelson DR, Nelson KA, Nixon K, Nusskern DR, Pacleb JM, Palazzolo M, Pittman GS, Pan S, Pollard J, Puri V, Reese MG, Reinert K, Remington K, Saunders RD, Scheeler F, Shen H, Shue BC, Sidén-Kiamos I, Simpson M, Skupski MP, Smith T, Spier E, Spradling AC, Stapleton M, Strong R, Sun E, Svirskas $R$, Tector $C$, Turner $R$, Venter $E$, Wang $A H$, Wang $X$, Wang $Z Y$, Wassarman 
DA, Weinstock GM, Weissenbach J, Williams SM, Woodage T, Worley KC, Wu D, Yang S, Yao QA, Ye J, Yeh RF, Zaveri JS, Zhan M, Zhang G, Zhao Q, Zheng L, Zheng XH, Zhong FN, Zhong W, Zhou X, Zhu S, Zhu X, Smith HO, Gibbs RA, Myers $\mathrm{EW}$, Rubin GM, Venter JC. The genome sequence of Drosophila melanogaster. Science 2000;287:2185-95.

16 Yeo G, Burge CB. Maximum entropy modeling of short sequence motifs with applications to RNA splicing signals. J Comput Biol 2004;11:377-94.

17 Khan AO, Bolz HJ, Bergmann C. Results of fibrillin-1 gene analysis in children from inbred families with lens subluxation. J AAPOS 2014;18:134-9.

18 Hilhorst-Hofstee Y, Rijlaarsdam ME, Scholte AJ, Swart-van den Berg M, Versteegh MI, van der Schoot-van Velzen I, Schäbitz H-J, Bijlsma EK, Baars MJ, Kerstjens-Frederikse WS, Giltay JC, Hamel BC, Breuning MH, Pals G. The clinical spectrum of missense mutations of the first aspartic acid of cbEGF-like domains in fibrillin-1 including a recessive family. Hum Mutat 2010;31: E1915-27.
19 Hogue J, Lee C, Jelin A, Strecker MN, Cox VA, Slavotinek AM. Homozygosity for a FBN1 missense mutation causes a severe Marfan syndrome phenotype. Clin Genet 2013;84:392-3.

20 Van Dijk FS, Hamel BC, Hilhorst-Hofstee Y, Mulder BJM, Timmermans J, Pals G, Cobben JM. Compound-heterozygous Marfan syndrome. Eur J Med Genet 2009;52:1-5.

21 Palz M, Tiecke F, Booms P, Göldner B, Rosenberg T, Fuchs J, Skovby F, Schumacher H, Kaufmann UC, von Kodolitsch Y, Nienaber CA, Leitner C, Katzke S, Vetter B, Hagemeier C, Robinson PN. Clustering of mutations associated with mild Marfan-like phenotypes in the $3^{\prime}$ region of FBN1 suggests a potential genotype-phenotype correlation. Am J Med Genet 2000;91:212-21.

22 Milewicz DM, Grossfield J, Cao SN, Kielty C, Covitz W, Jewett T. A mutation in FBN1 disrupts profibrillin processing and results in isolated skeletal features of the Marfan syndrome. J Clin Invest 1995;95:2373-8.

23 Buoni S, Zannolli R, Macucci F, Ansaldi S, Grasso M, Arbustini E, Fois A. The FBN1 (R2726W) mutation is not fully penetrant. Ann Hum Genet 2004;68(Pt 6):633-8. 